\title{
Norois
}

Environnement, aménagement, société

$212 \mid 2009 / 3$

Gestion urbaine et inégalités socio-spatiales France, Brésil

\section{Les logiques sécuritaires dans le discours des promoteurs et des résidants des ensembles résidentiels fermés : l'exemple de Nantes}

Security logics in property developers and dwellers' discourses within fenced and protected residence: the example of Nantes

\section{François Madoré et Fanny Vuaillat}

\section{(2) OpenEdition}

Journals

\section{Édition électronique}

URL : https://journals.openedition.org/norois/2937

DOI : $10.4000 /$ norois.2937

ISBN : 978-2-7535-1560-4

ISSN : $1760-8546$

\section{Éditeur}

Presses universitaires de Rennes

Édition imprimée

Date de publication : 1 décembre 2009

Pagination : $9-22$

ISBN : 978-2-7535-1034-0

ISSN : 0029-182X

\section{Référence électronique}

François Madoré et Fanny Vuaillat, «Les logiques sécuritaires dans le discours des promoteurs et des résidants des ensembles résidentiels fermés : l'exemple de Nantes », Norois [En ligne], 212 | 2009/3, mis en ligne le 01 décembre 2011, consulté le 14 janvier 2022. URL : http://journals.openedition.org/ norois/2937 ; DOI : https://doi.org/10.4000/norois.2937 


\title{
LES LOGIQUES SÉCURITAIRES DANS LE DISCOURS DES PROMOTEURS ET DES RÉSIDANTS DES ENSEMBLES RÉSIDENTIELS FERMÉS : L'EXEMPLE DE NANTES
}

\author{
François Madoré, Fanny Vuaillat \\ CESTAN - ESO UMR 6590 CNRS \\ (Université de Nantes), \\ Chemin de la Censive-du-Tertre, BP 81227 - 44312 NANTES cedex 3, France \\ francois.madore@univ-nantes.fr,fanny.vuaillat@univ-nantes.fr
}

\section{RÉSUMÉ}

Les logiques sécuritaires participent-elles de la construction de l'argumentaire légitimant la multiplication des dispositifs de fermeture de l'espace résidentiel en France? L'analyse des représentations du phénomène, basée sur l'approche discursive (seize entretiens de promoteurs français et vingt auprès d'habitants de deux résidences closes et sécurisées de l'agglomération nantaise) permet de répondre à la question. Quatre idées fortes ressortent: le consensus ou l'indifférence autour de la fermeture résidentielle dans les discours, la banalisation sécuritaire dans les discours promotionnels, un rapport ambigu à la fermeture et à la sécurisation dans les discours des résidants, enfin l'absence d'illusion sur l'efficacité de la sécurisation dans les discours.

MoTS CLÉ : résidence fermée - représentations - logiques sécuritaires - promoteur - habitant - discours - France - Nantes

\section{ABSTRACT}

Security logics in property developers and dwellers' discourses within fenced and protected residence: the example of Nantes

Do security logics take part of the selling arguments legitimating the multiplication of fencing and protection devices in residential areas across France? An analysis of the representations of the phenomenon, out of an interviewed-based approach (sixteen in-depth interviews with French property developers and twenty ones with dwellers of two fenced and protected residences in the Greater Nantes Area), makes some answers possible to this question. Four main points arise: consensus or indifference about residential fencing, security evidence in the property developers' arguments, ambiguous relations with fencing and security within dwellers' discourses and at last, lack of illusion regarding the effectiveness of the security approach.

KEY WORDS : fenced and protected residence - representations - security logics - property developers - dwellers - discourses - France - Nantes 
La connaissance de la fermeture résidentielle, tant dans son amplitude géographique que dans ses modalités de fonctionnement, a beaucoup progressé ces dernières années grâce à l'organisation des colloques bi-annuels du réseau de recherche internationale sur les gated communities $^{1}$ et à la floraison de publications traitant du sujet. Nous savons désormais que les ensembles résidentiels fermés se déploient un peu partout dans le monde (Billard et al., 2005 ; Glasze et al., 2005; Le Goix, Webster, 2008; Paquot, 2009), même si la couverture spatiale du phénomène reste encore très inégale. Le recensement effectué en 2007 à l'échelle du territoire français, grâce à l'exploration des sites Internet des promoteurs, illustre cette banalisation du phénomène (Billard et al., 2009²). Ainsi, les sièges sociaux des 123 promoteurs affichant sur leur site Internet au moins une opération immobilière fermée appartiennent à dix-huit régions sur vingt-trois (l'outre mer a été globalisé en une seule entité). Par ailleurs, la diffusion ubiquiste des programmes fermés avec contrôle des accès est très nette : des opérations sont localisées dans la quasi-totalité des régions françaises, soit 22 (21 en métropole plus l'Outre Mer) sur 23. Toutefois, même si le phénomène s'est banalisé, il reste comme en 2002, date du précédent recensement (Madoré, 2003), à la fois discriminé géographiquement, marquant de son empreinte les régions et les villes situées principalement au sud de la France puis à l'est, et divers dans son expression. Certes, certains cas sont plus fréquents que d'autres, avec comme figure pivot l'ensemble d'habitat collectif fermé par un grillage doublé d'une haie dont le contrôle des accès est assuré par un portail automatique, mais autour de cette figure centrale, les déclinaisons sont nombreuses, que l'on prenne le type d'habitat, de standing, de population, d'enclosure ou les modalités de contrôle des accès et de surveillance.

La plupart des travaux portant sur la fermeture résidentielle évoquent de façon plus ou moins explicite l'existence d'une articulation entre ce phénomène et la montée de l'insécurité réelle ou perçue et des discours sur l'insécurité. Certes, ce lien n'épuise pas la diversité des processus à l'œuvre, mais il est fréquemment appelé à la rescousse des facteurs explicatifs. Par ailleurs, la logique sécuritaire s'exprime selon des intensités très variables géographiquement et cette référence commune n'est pas systématiquement porteuse de la même signification d'un contexte spatial à l'autre. Il est de fait plus conforme à la réalité d'évoquer ces logiques sécuritaires au pluriel. Celles-ci oscillent entre la peur de la criminalité sous différentes formes (atteinte aux personnes et aux biens) et celle de l'Autre, les deux pouvant d'ailleurs être confondues, mais aussi entre la recherche de tranquillité et la volonté de privilégier des formes variées d'entre soi, moyen de conjurer certaines craintes et de gérer ainsi le risque lié à ces craintes.

Ce sont ces logiques sécuritaires que nous souhaitons interroger, pour voir comment elles participent ou non de la construction de l'argumentaire légitimant la multiplication des dispositifs de sécurisation et de fermeture de l'espace résidentiel en France. Une des pistes les plus prometteuses en termes d'approfondissement de cette question porte sur l'analyse des représentations du phénomène. Dans cette logique, deux acteurs ont été investigués : les promoteurs et les résidants. Interroger les premiers permet de comprendre les logiques d'offre à l'origine de ces nouveaux modes d'habitat constitutifs de l'enclosure résidentielle, pourquoi et comment les promoteurs intègrent la clôture mais aussi le contrôle des accès dans la conception de leur programme. Interviewer les seconds permet de saisir les raisons de leur installation dans de tels complexes, mais aussi plus globalement la représentation qu'ils s'en font et la façon dont ces modes d'habitat sont susceptibles d'influer sur leurs pratiques de l'espace. Les représentations des résidants des complexes fermés ou sécurisés sont d'autant plus intéressantes à observer que ce type d'acteur avait rarement été questionné jusqu'à ces toutes dernières années, que ce soit en France ou dans d'autres contextes géographiques. Néanmoins, cette situation évolue, comme en témoigne la présentation d'un

1. Adresse Internet, [www.gated-communities.de].

2. Ce recensement a été réalisé au cours de la première phase d'un programme de recherche financé par l'INHES (Institut national des hautes études de sécurité) et intitulé Typologie et représentations des ensembles résidentiels fermés ou sécurisés en France (Madoré, 2008). Six chercheurs ont travaillé sur ce programme : G. Billard, J. Chevalier, F. Madoré, F. Raulin, A. Taburet, F. Vuaillat. 
certain nombre de recherches qui toutes justifient le bien-fondé de cette approche, qu'il s'agisse des travaux dirigés par G. Capron (2006) sur l'Amérique latine, ceux conduits sur une résidence fermée située à Toulouse (Golovtchenko et Souchet, 2005) et à Nantes (Vuaillat et Madoré, 2008), ou encore un certain nombre de communications présentées au quatrième colloque du réseau de recherche internationale sur les gated communities ${ }^{3}$ organisé à Paris en juin 2007.

L'intérêt de cette double démarche conduite auprès des promoteurs et des résidants est précisément de croiser les regards, de confronter les deux types de discours, en particulier les fragments de discours des promoteurs relatifs aux attentes de la clientèle en matière de sécurité et les représentations de cette dernière. Après une présentation de la méthodologie mise en œuvre et des deux résidences enquêtées à Nantes, trois idées fortes ressortent à l'examen de la confrontation des discours tenus à la fois par les promoteurs et les résidants. La première pointe l'indifférence des acteurs interrogés face au développement des ensembles résidentiels fermés. La seconde relève une discordance entre les discours des promoteurs et des résidants quant à la place réservée à la variable sécuritaire dans l'argumentaire justifiant le développement de la fermeture et du contrôle des accès. Quant à la troisième, elle souligne l'absence d'illusion sur l'efficacité de la sécurisation dans les discours tenus tant par les résidants que par certains promoteurs.

\section{Méthodologie : entretiens et analyse discursive}

Une différence fondamentale oppose les discours des promoteurs et ceux des résidants. La saturation du discours est atteinte beaucoup plus rapidement chez les premiers, car les variations inter-discours sont plus réduites. Les discours sont également peu discriminés territorialement, la plupart des promoteurs interrogés affirmant clôturer et imposer le contrôle des accès à leur programme quelle que soit sa localisation. Tout au plus, certains concèdent une plus grande fréquence et une antériorité de la fermeture à la fois en région parisienne et sur la Côte d'Azur. À l'inverse, les variations inter-discours sont beaucoup plus prononcées chez les résidants, ce qui n'est guère surprenant. Celles-ci tiennent à la fois à la diversité des effets de contexte géographique, aux poids des déterminants sociodémographiques des individus, à leur vécu et aux représentations qu'ils ont développées. C'est pourquoi, tirer des traits généraux en termes d'analyse de la succession des discours des habitants peut constituer un exercice périlleux et très aléatoire. Afin d'éviter cet écueil, il est dès lors impératif de maîtriser les effets de contexte, car les fragments de discours analysés ne valent que dans un contexte donné. C'est pourquoi, le regard des promoteurs français sur la fermeture résidentielle va être confronté à celui des résidants de deux contextes d'habitat différents mais situés l'un comme l'autre dans l'agglomération nantaise.

Au niveau des promoteurs, le corpus discursif analysé est composé de seize entretiens, dix conduits en 2002 et six fin 2007-début 2008. Au sein de ces groupes de promotion immobilière, les interlocuteurs ont été, généralement, le responsable des programmes ou le directeur technique, voire dans les plus petites sociétés, le directeur lui-même. Notre choix a été de constituer l'échantillon le plus représentatif possible de la diversité des sociétés de promotion immobilière à l'œuvre en France. Il croise ainsi des promoteurs de taille très variable, depuis des petites sociétés jusqu'aux majors de la profession. Par ailleurs, si certains ont une activité relativement généraliste, s'adressant à une clientèle aussi bien constituée d'accédants que d'investisseurs, d'autres en revanche sont des spécialistes de l'investissement immobilier locatif défiscalisé. Au total, quinze promoteurs ont été interrogés, car l'un, à savoir le même interlocuteur, l'a été à la fois en 2002 et en 2007, ce qui nous a confirmé l'invariabilité du discours à cinq ans d'intervalle. Ces promoteurs sont

3. $4^{\text {th }}$ international conference of the research network Private urban governance and gated communities, Private urban governance: Production of urban space, Interactions of public and private actors, Sustainability of cities, [www.gatedcommunities.de]. 
basés dans quatre aires géographiques distinctes : six ont leur siège social en région parisienne ${ }^{4}$, six autres à Toulouse ${ }^{5}$, deux en Martinique ${ }^{6}$ et enfin un à Nantes (il s'agit en fait de la direction régionale d’un promoteur national : Bouygues Immobilier région Atlantique).

Ce matériau discursif est confronté à celui constitué par le recueil de la parole habitante dans deux résidences closes et sécurisées situés dans l'agglomération nantaise. Vingt entretiens ont ainsi été réalisés auprès d'habitants : dix à la résidence Le Newton à Carquefou (photo 1) dans la banlieue de Nantes (en janvier 2006) et dix autres au Domaine de la Guylhomné (photo 2) sur la commune de Nantes (en mars 2008). Parallèlement, deux entretiens ont été passés auprès d'acteurs jouant un rôle central dans le fonctionnement de ces deux copropriétés, à savoir le gardien régisseur dans la première et le président du conseil syndical dans la seconde. En général, les entretiens auprès des résidants ont duré entre 20 et 45 minutes et se sont tous déroulés au domicile des enquêtés. L'entretien semi-directif est apparu comme la méthode idoine pour faire émerger les discours d'existence, donnant de la sorte accès aux images et aux représentations des habitants dans la construction de leur rapport à l'habiter.

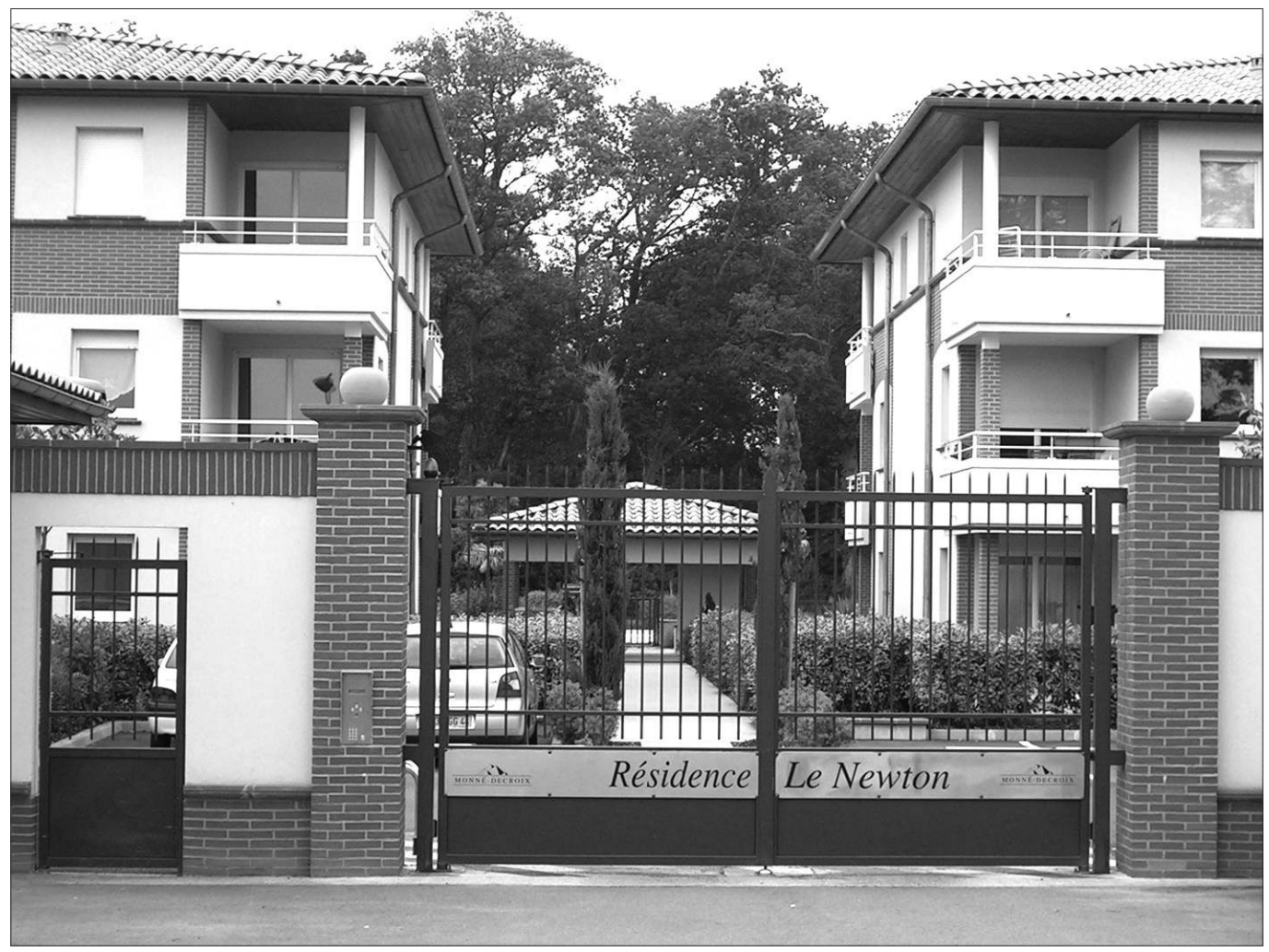

Photo 1 : Le Newton (cl. F. Vuaillat)

The Newton property

4. Bouygues Immobilier, Eiffage Immobilier, Espace 2, European Homes, les Nouveaux Constructeurs et Promogim.

5. Akerys, Fonta, LP Promotion, Malardeau, Monné Decroix et Sagec. Le choix d'interroger les promoteurs toulousains s'explique doublement : d'une part, ce sont parmi les premiers, a priori, à avoir intégré en France l'objectif de la clôture dans leurs programmes résidentiels; d'autre part, la capitale de Midi-Pyrénées occupe en 2007, et de très loin, la première place en province pour la concentration de promoteurs affichant des programmes clôturés avec contrôle des accès.

6. Maisons Beterbat et Sibat. 


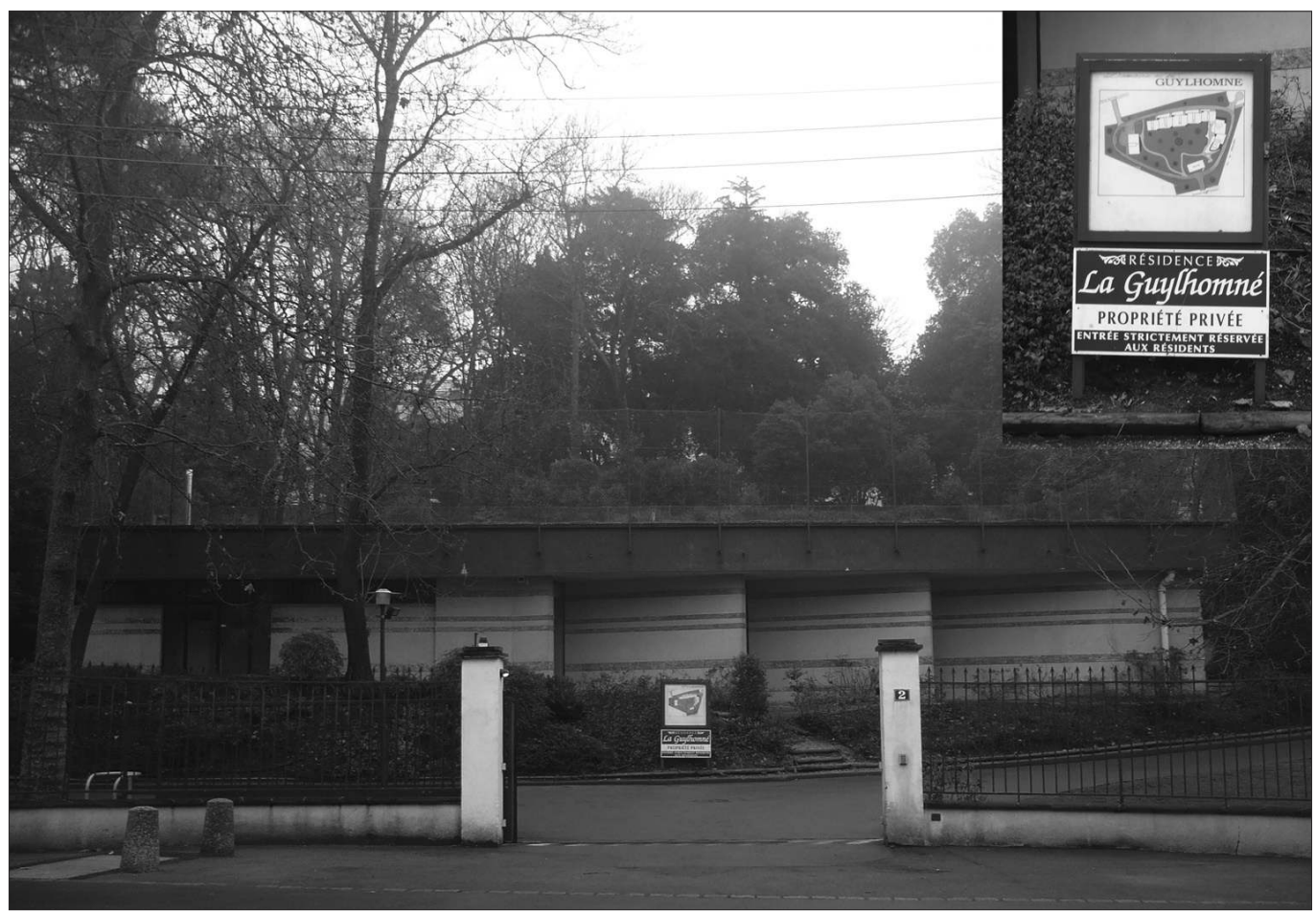

Photo 2 : Le Domaine de la Guylhomné (cl. F. Vuaillat) The Guylhomné property

L'une des difficultés de mise en œuvre de cette méthodologie dans des contextes marqués par la fermeture résidentielle est précisément l'accès aux deux résidences. Au Domaine de la Guylhomné, la prise de contact s'est faite par l'intermédiaire du gardien, qui nous a orientés vers le président du conseil syndical. Celui-ci nous a autorisés à nous présenter auprès des résidants en son nom. Pour commencer, il nous a dirigés vers sa belle-mère, résidante de la tour. Puis, le contact avec les autres habitants s'est effectué soit par le porte-à-porte, soit par les relations de voisinage des personnes entretenues. Au Newton, pour pouvoir nous entretenir avec des habitants, il nous a fallu au préalable demander une autorisation auprès du directeur commercial de l'agence Monné Decroix de Nantes, qui gère cette résidence. Celui-ci a prévenu le gardien régisseur qui a affiché notre passage dans les cages d'escalier. Nous avons donc rencontré ce gardien qui nous a transmis les codes d'entrée et de sortie, nous autorisant à circuler à notre guise au sein de la résidence. Puis, le contact avec les résidants s'est effectué principalement par le porte-à-porte.

\section{Présentation des deux résidences enquêtées à Nantes}

Étant donné la différence de nature évoquée entre les entretiens promoteurs et ceux réalisés auprès de résidants, la maîtrise des effets de contexte résidentiel est nécessaire et indispensable pour apprécier les résultats de l'analyse discursive. Il convient donc de présenter ces contextes, donc les deux résidences enquêtées. 
François Madoré, Fanny Vuaillat

\section{Le Domaine de la Guylhomné}

Le Domaine de la Guylhomné est situé dans les beaux quartiers péricentraux nantais de Procé, à l'ouest du cœur historique. La résidence se trouve dans une ancienne propriété, au milieu d'un parc de presque deux hectares, au sein duquel a été réalisée, entre 1967 et 1968, une opération immobilière d'envergure : la construction de deux tours de douze et dix étages comprenant au total environ 150 logements (du T1 ou T7). Cette résidence possède par ailleurs un certain nombre d'aménités : une piscine couverte, un court de tennis et une salle de réception. Les résidants sont à $90 \%$ propriétaires de leur logement. Une bonne partie d'entre eux habitant la résidence depuis sa construction, leur âge moyen est donc élevé. D’autre part, le prix d'acquisition d'un appartement étant particulièrement cher $\left(5000 €\right.$ au $\mathrm{m}^{2}$ en mai 2007 , sachant par ailleurs que les charges mensuelles s'élèvent pour un T4 à environ $400 €$ ), les résidants appartiennent en majorité aux catégories aisées.

En termes de modalités de fermeture et de sécurisation de l'espace, le Domaine de la Guylhomné est ceint d'un mur, correspondant à celui de l'ancienne propriété. À l'origine, ce mur avait des tailles différentes (entre un et trois mètres), mais l'installation de grilles ou de grillages a permis une uniformisation relative des hauteurs de l'enclosure (entre deux et trois mètres aujourd'hui). Le contrôle des accès à l'entrée de la résidence a été instauré en 1994, avec la pose d'un portail automatique au niveau de l'entrée principale. Celui-ci reste cependant ouvert de 7 heures à 9 heures et de 17 heures à 19 heures la semaine et en dehors des vacances scolaires. Deux entrées secondaires sont quant à elles fermées en permanence. Par ailleurs, trois caméras de vidéosurveillance filment 24 heures sur 24 l'entrée principale, la piscine et une partie du parking la plus en vue de l'extérieur. Seul le président du conseil syndical est homologué à visionner les enregistrements. Ces caméras ont été installées au début des années deux mille et ont remplacé la ronde qu'effectuait deux fois par semaine une société de sécurité, rondes jugées inefficaces et coûteuses. Enfin, un gardien est présent sept heures par jour en semaine dans la résidence, remplaçant depuis quinze ans, toujours pour des questions de budget, deux couples de gardien à plein-temps qui officiaient auparavant.

\section{LA RÉSIDENCE LE NEWTON}

La résidence le Newton est située à Carquefou, dans la banlieue nord de Nantes, au sein de la ZAC de la Fleuriaye. C'est une résidence locative construite au début des années deux mille et gérée par le groupe Monné Decroix basé à Toulouse. Elle regroupe quatre petits collectifs $(\mathrm{R}+2)$, soit 90 logements du type 1 au type 4. La résidence est dotée comme celle de la Guylhomné d'aménités, avec une piscine extérieure ouverte du $1^{\text {er }}$ juin au $1^{\text {er }}$ septembre et un parc paysager soigné et propre, d'où des restrictions d'usage pour maintenir cet état de propreté : les animaux domestiques, les repas ou les fêtes y sont interdits. Le complexe profite par ailleurs de la grandeur des vieux arbres environnants et de l'image verdoyante du site. Les résidants sont majoritairement jeunes (entre 25 et 45 ans), la plupart étant en période de transition, soit à la suite de difficultés (désunion, chômage, etc.), soit en cours de réalisation de projets (construction de maison, création ou reprise d'entreprise, adoption, etc.). Du fait des contraintes liées à la loi de défiscalisation, qui imposent un plafonnement des revenus pour les locataires, mais aussi de la taille des appartements et du prix des loyers (de $351 €$ charges comprises pour un T1 à $883 €$ pour un T4, ce qui correspond au prix du marché, voire en deçà si l'on considère les aménités de la résidence), les résidants sont représentatifs des " classes moyennes inférieures ».

Les modalités de fermeture et de contrôle des accès sont marquées à la fois par l'enclosure totale de la résidence, à l'aide d'un grillage doublé d'une haie végétale, et par la présence d'un double portail automatique, l'un pour les piétons, l'autre pour les véhicules. À ce premier contrôle des accès s'en surimpose un second à l'entrée de chaque immeuble, à l'aide d'une clé pour les résidants ou d'un interphone pour les visiteurs. De surcroît, un dispositif de vidéosurveillance composé de cinq caméras permet de visualiser à toute heure l'entrée principale du complexe, 
ainsi que les entrées de chaque immeuble. La visualisation se fait à l'aide des postes de télévision de chaque résidant sur un canal spécifique. Outre la vidéosurveillance, chaque appartement est muni d'un système d'alarme. Enfin, la présence d'un gardien contribue aussi à la surveillance du complexe résidentiel, du moins en journée, car le gardien ne réside plus au Newton depuis son déménagement en novembre 2005.

\section{Consensus ou indifférence autour de la fermeture résidentielle dans les discours}

La large diffusion d'une nouvelle territorialité de l'habiter en France construite sur le mode de la fermeture et du contrôle des accès semble faire consensus, ou peu s'en faut. Les promoteurs estiment qu'il n'est guère possible désormais de construire autrement, car cela ne « serait pas dans l'air du temps ». Quant aux résidants interrogés dans les deux complexes résidentiels nantais, ils semblent accepter sans coup férir la fermeture et le contrôle des accès, à la fois parce qu'ils ne sont pas réellement en mesure de peser sur les choix conceptuels des nouveaux espaces d'habiter, mais aussi parce que nombre d'entre eux ne s'impliquent que très peu dans la gestion de leur copropriété une fois celle-ci constituée, abandonnant ainsi à une minorité de copropriétaires et au syndic le réel pouvoir décisionnel.

L'exemple du Domaine de la Guylhomné dans les beaux quartiers péricentraux nantais, résidence qui a connu ces dernières années un mouvement de sécurisation (cf. supra), illustre bien les limites de la participation au processus décisionnel, alors même qu'elle est habitée essentiellement par des propriétaires occupants, dans la mesure où les décisions sont prises en réalité par la douzaine de personnes en charge du conseil syndical. De fait, la tonalité dominante en termes de discours relatif à la sécurisation et au contrôle des accès est empreinte d'une forte dose de fatalisme. En clair, les habitants ont peu d'avis sur la question et n'ont, pour la plupart, pas pris part aux discussions. Lorsqu'ils émettent une opinion, celle-ci se résume la plupart du temps à l'expression suivante : la sécurisation des espaces est un mouvement général considéré comme normal, voire parfois nécessaire.

\section{Extraits d'entretien - Le Domaine de la Guylhomné}

- On est obligé de le faire, mais je crois que c'est comme partout, ce n'est pas seulement ici.

- Avant c'était plus ouvert, maintenant tout est fermé, bloqué. Je ne sais pas si vous vous êtes promené dans la résidence, mais les parkings et tout, tout est clos, à clé, il faut vraiment... ils ont tout verrouillé. C'est qu'il y avait peut-être besoin. Voilà.

- Il y a tellement de malheurs à gauche et à droite, des violences, des effractions, je pense que le conseil syndical s'est méfié et a pris peut-être un peu trop de précaution.

- On est obligé de suivre l'air du temps, on ne peut pas dire autre chose. C'est comme ça.

\section{Banalisation sécuritaire dans les discours promotionnels}

Pour les promoteurs interrogés, sans exception, la mise en œuvre de la clôture et du contrôle des accès dans leurs programmes immobiliers est une réponse à la demande exprimée par la clientèle. Toutefois, cette demande est rarement connue avec précision. Elle est soit appréhendée de façon empirique, à partir du ressenti des commerciaux, soit le fruit du constat suivant : puisque les programmes immobiliers fermés se vendent bien, c'est donc qu'ils rencontrent les attentes de la clientèle. Mais quelle est réellement la marge de manœuvre du client potentiel, dès lors que la fermeture et le contrôle des accès ont tendance à se banaliser? Le client est-il en position de choix? Au-delà de ces incertitudes relatives à la connaissance réelle de la demande de la clien- 
tèle, que nous apprend le discours des promoteurs sur ce que rechercherait le client souhaitant acquérir un logement?

La construction d'un discours promotionnel légitimant la fermeture et le contrôle des accès dans l'habitat se fonde pour partie, en France, sur la nécessité de se protéger de la criminalité. Cette invasion du discours faisant référence de façon très explicite à l'insécurité chez les promoteurs immobiliers a pour conséquence, entre autres, une banalisation de la rhétorique sécuritaire dans la rédaction des descriptifs des programmes immobiliers neufs, qu'ils soient clôturés ou non d'ailleurs. Ainsi, les promoteurs interrogés estiment tout à fait inconcevable aujourd'hui de construire un programme résidentiel sans un contrôle des accès et sans une panoplie d'éléments de sécurisation.

\section{Extraits d'entretien}

\section{Akerys (Toulouse)}

La tendance est à la sécurité [...] on ne peut pas accepter qu'une porte soit ouverte, que les gens circulent comme ça. Donc je m'aperçois que nos programmes réalisés avec clôture se louent mieux que sans clôture. Donc, on répond à la demande, on ne fait que répondre à la demande.

Eiffages (Paris)

Aujourd'hui, la demande d'un client, elle est quand même plutôt sécuritaire et on se voit pas proposer un logement qui n'ait pas au moins un digicode extérieur sur une première porte de sas et puis, a minima, un interphone après, et puis donc une grille de clôture pour fermer [...]. Si effectivement un client avait le choix entre deux types de promoteurs, je pense qu'il va incliner favorablement vers celui qui aura mis un accent particulier sur ce domaine de la sécurité, ça c'est clair.

\section{LP Promotion (Toulouse)}

C'est à la demande de tous nos clients, parce que de plus en plus il y avait un peu de délinquance et les gens voulaient se sentir en sécurité dans leur résidence [...]. Et ça devient un point très important [...] Les gens sont très sensibles à l'heure actuelle à la sécurité.

\section{Monné Decroix (Toulouse)}

La sécurité, c'est un argument [...] il ne faut pas se leurrer, aujourd'hui c'est un atout, un argument de location et de vente. Ça ne l'était pas, mais les faits de société font que ça l'est devenu [...] Oui, oui, il y a une attente, donc il faut répondre à cette attente.

\section{Promogim (Paris)}

C'est un lieu commun aujourd'hui de parler de la sécurité. La plupart des gens souhaitent être à l'abri le plus possible [...]. L'opération close avec un contrôle d'accès [...] ça les acquéreurs sont très très demandeurs.

Les modalités de sécurisation des programmes résidentiels et du contrôle des accès proposés par les promoteurs sont doubles :

- Un premier filtre est situé au niveau de l'accès au complexe résidentiel depuis la rue. Cela passe par un arsenal technologique désormais classique : portail ou portillon automatique avec code d'accès ou badge pour les résidants (à l'image du système Vigik où un badge est attribué aux résidants mais aussi aux concessionnaires ou prestataires de services autorisés, comme les agents de la Poste ou d'EDF par exemple) puis, pour les visiteurs, interphone, digicode et, de plus en plus, visiophone. Plus exceptionnellement, un système de vidéosurveillance peut-être mis en place.

- Certains promoteurs installent un deuxième filtre, à l'aide d'un interphone, digicode ou visiophone, à l'intérieur même du complexe résidentiel, entre les parties communes et les immeubles, de telle sorte qu'un intrus ne puisse pas pénétrer au sein des immeubles s'il avait réussi à franchir l'entrée du complexe. La sécurisation et le contrôle des accès au Newton sont conçus sur ce principe (cf. supra). Selon la même logique, des filtres avec code d'accès peuvent être placés entre les différentes parties communes d'un immeuble, toujours avec le même objectif, à savoir limiter la 
mobilité d'un intrus en le confinant dans une zone. Ainsi, des codes d'accès peuvent être installés pour passer du parking souterrain aux étages, via l'ascenseur ou la cage d'escalier.

Tous ces filtres, qu'ils soient situés au niveau de l'interface espace public/complexe résidentiel ou à l'intérieur de celui-ci, participent du même objectif : empêcher ou dissuader l'intrusion ou, si celle-ci a eu lieu malgré tout, multiplier les contraintes pour limiter les mouvements d'un intrus.

\section{Rapport ambigu à la fermeture et à la sécurisation dans les discours des résidants}

\section{Assurer la TRANQUILLITÉ ET L'EXCLUSIVITÉ EN TERMES DE FRÉQUENTATION DES AMÉNITÉS}

Si le discours promotionnel légitime pour partie l'essor de la fermeture résidentielle et du contrôle des accès à l'entrée des complexes d'habitat par la nécessité de se protéger de la criminalité, les discours tenus par les résidants interrogés à Nantes sont relativement divers et paradoxaux, révélant un certain décalage avec le discours relativement uniforme des promoteurs. Les variabilités inter-discours sont ainsi très fortes, avec un spectre allant de l'appréciation des dispositifs sécuritaires à leur rejet, en passant par l'indifférence ou un regard plutôt distancié. Les discours positifs émanent pour l'essentiel de résidants âgés du Domaine de la Guylhomné, qui peuvent se sentir en situation de vulnérabilité physique, d'où une sensibilité accrue à tout ce qui touche à la sécurité, aussi bien personnelle que celle de leurs biens. Mais quelques habitants du Newton ont également exprimé cette sensibilité.

Toutefois, cette appréciation positive des dispositifs de sécurité ne signifie pas, loin s'en faut, une sensibilité particulière et exclusive à la sécurité des personnes et des biens. Ainsi, pour certains résidants interviewés, ce regard positif est autant sinon plus motivé par une volonté de préserver sa tranquillité en mettant à distance l'intrus, quel qu'il soit. Cette quête de tranquillité rejoint d'autres observations, comme celles réalisées par É. Charmes (2005) dans des ensembles résidentiels du périurbain parisien et lyonnais. Pour d'autres résidants ou les mêmes, l'intérêt de la clôture et du contrôle des accès tient aussi à la possibilité de préserver l'exclusivité de la fréquentation des équipements collectifs comme la piscine, tout en protégeant la copropriété, qui serait responsable en cas d'accident à un tiers. En ce sens, le discours des résidants, du moins certains d'entre eux, rejoint sur ce registre celui des promoteurs, qui estiment que l'un des facteurs déterminants de la demande en faveur de nouveaux territoires résidentiels construits sur le mode de la fermeture est, outre la nécessité de se protéger des actes délictueux, le processus d'appropriation des espaces collectifs privatifs. En clair, il s'agit d'abord et avant tout de borner la propriété par l'imposition d'une limite claire. Ce processus réactive en fait une composante des modes d'habiter, dans la mesure où, en France, pour des raisons culturelles et urbanistiques, la fermeture de l'habitat est une pratique courante. Cette logique d'appropriation par la fermeture permet ainsi de conformer l'accessibilité du domaine résidentiel à son statut juridique, ce qui renvoie aussi à la notion de tranquillité. Ce souci de conformer l'usage du lieu à son statut est d'autant plus marqué que le complexe résidentiel possède un certain nombre d'aménités, généralement un espace vert et une piscine, ce qui est le cas au Domaine de la Guylhomné et au Newton.

\section{Extraits d'entretien}

Le Domaine de la Guylhomné - Certaines personnes de l'immeuble filtrent beaucoup à l'interphone pour empêcher les gens extérieurs

d'entrer dans les escaliers.

- Il faut se protéger, oui, se protéger.

- C'est sûr que l'aspect sécurité est important. 


\section{La Résidence Le Newton}

- Je sais que l'on peut visualiser les caméras sur la télévision, ça c'est génial.

- Une fois une personne devait passer et je ne voulais pas, c'était pour me vendre des trucs et donc là j'ai regardé et j'ai vu.

- Ici, c'est vraiment sécurisé et anjourd'hui je trouve que c'est hyper important de se sentir à l'aise, de ne pas être tout le temps stressé à regarder par la fenêtre pour voir si quelqu'un n'est pas en train de vous forcer votre voiture.

\section{DisCourS CRITIQUE ET GÈNE OCCASIONNÉE PAR LE CONTRÔLE DES ACCÈS}

À l'antithèse de cette appréciation des dispositifs de sécurisation et de contrôle des accès, émerge un discours beaucoup plus critique sur la fermeture et l'abondance de l'artillerie sécuritaire. Ces fragments de discours sont en totale déphasage avec le ressenti des promoteurs. Cette appréciation assez négative repose pour l'essentiel sur la gêne occasionnée dans la pratique quotidienne de l'espace résidentiel. La plupart des habitants, dans les deux résidences enquêtées, ont ainsi exprimé des plaintes. Certaines personnes âgées par exemple, majoritaires au Domaine de la Guylhomné, peuvent rencontrer des difficultés avec les diverses clés, portails et codes à retenir. La fermeture semble poser certains problèmes aussi aux enfants qui, oubliant leurs clés, ne peuvent entrer dans la résidence. L'imposition d'un voire de deux contrôles des accès avant de pouvoir pénétrer dans l'immeuble complique également la réception d’invités. Enfin, certains résidants du Newton déclarent être l'objet de railleries de la part des personnes extérieures, qui évoquent l’univers carcéral pour décrire leur espace résidentiel.

Ce sont les habitants du Newton qui adoptent la posture la plus critique vis-à-vis des dispositifs de sécurité, considérant même «l'artillerie sécuritaire » comme un gadget commercial du groupe Monné Decroix. D'ailleurs, ils ne se gênent pas pour rappeler les nombreux dysfonctionnements de ces dispositifs, liés selon eux à leur mauvais entretien par les gestionnaires. Toutefois, cet état déficient des équipements de sécurité ne génère pas d’inquiétude, dans la mesure où ils sont perçus comme des gadgets à l'efficacité limitée.

\section{Extraits d'entretien}

Le Domaine de la Guylhomné

- C'est vrai que maintenant on se retrouve avec des portes, des clés, des codes, c'est vrai que ça devient complètement fou quand on a plusieurs codes en tête, alors l'ouverture des portes!

- On a tout fait, c'est à un point que l'on ne peut plus entrer, ni sortir si on a oublié ses clés, c'est épouvantable.

- Bon, il y a eu un ou deux malheurs en 40 ans mais pas plus et là tout d'un coup c'est vraiment une astreinte.

- On descend les poubelles, il faut les poser, il faut sortir sa clé, ouvrir la porte, poser la poubelle, refermer la porte, en ouvrir une autre. Non, pour moi, c'est une contrainte. Il y a des tas de gens qui sont un peu contre d'ailleurs.

- C'est un peu exagéré, voilà, la sécurité est un peu exagérée.

- Ce qui est bizarre, c'est que l'on dit de ne pas donner le code, mais on est obligé de le donner quand quelqu'un vient, on ne va pas descendre lui ouvrir. Ce n'est pas possible.

- Et puis, le fait que ce soit fermé, il y a un petit côté ghetto, c'est vrai hein...

\section{La Résidence Le Newton}

- Il faudrait plus d'accessibilité pour les gens qui viennent nous voir.

- Il y a un côté, oui, enfermement qui est très pesant.

- Il faut les bips, on n'a pas le choix, c'était contraignant, enfin bon, ça permet aux gens d'en sourire, parfois d'en rire. 
- C'est ce côté ultra sécurisant que j'aimerais voir changer. Que les gens puissent entrer plus facilement et que nous nous puissions en sortir plus aisément sans passer par des sas.

- Tous nos copains qui sont venus nous ont dit «Mais t'es dans une prison, c'est quoi ce truc-là? »

- le bip, deux clés pour ouvrir, pour entrer, l'alarme, on se voit à la télé quand on rentre. Tout ça c'est des trucs qui font trop quoi.

- On est souvent comparé à vivre aux Baumettes ou à la Santé.

- La caméra infrarouge, quand on est arrivé, elle était en panne, elle n'a toujours pas été réparée, mais ça ne me gène pas. Je ne vais pas aller leur réclamer indéfiniment.

- La caméra elle ne sert à rien, elle n'enregistre pas.

\section{Absence d'illusion sur l'efficacité de la sécurisation dans les discours}

Quelle que soit l'appréciation portée par les résidants interrogés sur la fermeture, le contrôle des accès et la sécurisation de leur résidence, les discours tenus aussi bien au Domaine de la Guylhomné qu'au Newton témoignent de l'absence totale d'illusion sur l'efficacité des dispositifs de sécurisation pour prévenir des actes délictueux. Tout au plus, les habitants estiment que ces dispositifs sont de nature à se protéger des petites incivilités, comme l'utilisation des places de parking par des non résidants ou les déjections canines dans le parc. En guise d'illustration, depuis l'installation de la vidéosurveillance au Domaine de la Guylhomné, les auteurs de deux actes d'incivilités ont pu être identifiés : pour l'anecdote, les fautifs n'étaient pas extérieurs à la résidence! E. J. Blakely et M. G. Snyder (1997), dans leur enquête sur les gated communities étatsuniennes, avaient déjà souligné que la protection face à la délinquance, qui était l'un des moteurs du développement de cette forme d'habitat fermé, était toute relative, ne serait-ce que par le développement d'une petite criminalité de la part des adolescents inoccupés vivant à l'intérieur de ces ensembles.

\section{Extraits d'entretien}

\section{Le Domaine de la Guylhomné}

- La caméra c'est plus dissuasif qu'autre chose [...]. Vous savez, n'importe qui peut entrer.

- C'est un petit peu illusoire de vouloir fermer.

- Ça ne sera jamais un château fort, si les gens veulent vandaliser, ils le feront. Mais bon. Ça évite le simple curieux, celui qui veut entrer, il entre. Ce n'est pas très compliqué d'entrer.

\section{La Résidence Le Newton}

- C'est fermé sans être fermé, n'importe qui peut entrer.

- Les résidences protégées, c'est un peu utopique.

- Ils disent que c'est des résidences sécurisées, si t'as des barbelés autour, c'est pour que personne ne rentre et force est de constater qu'il suffit que quelqu'un ait ouvert, tu rentres et puis voilà.

Ce discours sans illusion sur l'efficacité réelle de la clôture et du contrôle des accès tenu par les résidants de la Guylhomné et du Newton rejoint le discours de certains promoteurs, qui évoquent l'aspect psychologique de la sécurisation mise en œuvre : à défaut d'offrir une garantie absolue ou même relative face aux actes délictueux, ces dispositifs rassurent les habitants. D'ailleurs, il peut paraître paradoxal d'observer d'un côté la banalisation de la rhétorique sécuritaire dans le discours promotionnel et, de l'autre, la reconnaissance, par la plupart des promoteurs interrogés, d'une absence d'éléments permettant d'apprécier correctement l'efficacité réelle de la clôture et du contrôle des accès. Peu importe en fait de savoir si réellement la clôture et le contrôle des accès sont bien dissuasifs en termes d'actes délictueux, l'important est d'en être convaincu. Cette remarque fait écho à celle soulignant l'absence de connaissance précise de la demande par les 
promoteurs, qui est généralement appréhendée de façon empirique, à partir du ressenti des commerciaux (cf. supra).

L'existence même de ce paradoxe montre à l'évidence que le discours promotionnel justifiant la fermeture par le recours à la rhétorique sécuritaire repose essentiellement sur une double médiatisation dans la construction des représentations de l'insécurité, à savoir la rumeur et la multiplication des discours insécuritaires. W. Ackermann, R. Dulong et H.-P. Jeudy (1983), dans leur analyse discursive de l'imaginaire véhiculé par l'insécurité, ont bien montré comment ce discours construit une rumeur. C'est donc en partie parce que l'insécurité, au-delà même de la réalité statistique du phénomène, préexiste à travers le discours et l'imaginaire, que la sensibilité des Français à l'insécurité est devenue si grande. Mais celle-ci a été largement alimentée par la multiplication des discours insécuritaires au sein de la sphère politico-médiatique (Mucchielli, 2001), sur fond de renouvellement progressif de la question de l'insécurité au cours des années soixante-dix (Robert, Pottier, 1997; Wievorka, 1999; Rey, 2002; Bonelli, 2008). Celle-ci intègre alors une nouvelle articulation, en interrogeant le lien entre délinquance et insécurité, au détriment de l'articulation qui dominait alors et qui associait violence politique et insécurité. Cette évolution, portée désormais aussi bien par la gauche que la droite, suite à l'aggiornamento de la gauche sur les questions de sécurité illustré par le colloque de Villepinte en 1997, où le gouvernement Jospin a consacré la sécurité grande cause nationale au même titre que la lutte contre le chômage, témoigne d'une appréhension globale de la question de l'insécurité, mettant sur un même pied d'égalité l'insécurité sociale et celle générée par des actes délictueux.

\section{Extraits d'entretien}

Akerys (Toulouse)

L'efficacité de la clôture est toute relative. Elle est d'abord psychologique.

Bouygues Immobilier (Paris)

Si on a la possibilité, on mettra des clôtures [...] qui ont deux rôles, je dirais plus un rôle d'abord esthétique, parce qu'en effet c'est plutôt joli, un rôle psychologique aussi, mais en termes d'efficacité pure, c'est très discutable.

\section{Bouygues Immobilier région Atlantique (Nantes)}

Question : est-ce que vous pensez que ce système de clôture, c'est vraiment efficace? — réponse : on n'a pas de retour, car on n'a pas de patrimoine à gérer... mais aussi a priori parce que ça se passe bien. On n'est pas dans une région à forte insécurité.

Malardeau (Toulouse)

Je pense qu'une simple clôture ça n'empêche pas les voleurs de passer [...]. Mais dans un but commercial, le client se sent sécurisé sur son produit.

Monné Decroix (Toulouse)

La clôture, c'est dissuasif, c'est une sécurité passive, et qui entend passive, dit en même temps effectivement dissuasion [...]. Je vous mets au défi de ne pas rentrer dans une de nos résidences [...] Ah oui, oui, on y rentre [...]. Il ne faut pas rêver, nos clôtures dans nos résidences, c'est entre guillemets de la dissuasion. Quelqu'un qui veut rentrer dans nos résidences, c'est très facile. Vous prenez une tenaille ou une pince, ou même vous attendez que quelqu'un rentre en voiture et puis vous suivez et puis voilà.

\section{Promogim (Paris)}

On sait bien que c'est illusoire, la sécurité. On ne peut pas prétendre que les gens ne seront pas cambriolés, qu'on n'ira pas voler quelque chose dans leur voiture, etc. bien entendu. Mais ça donne un gage minimum de protection [...]. Il y a effectivement l'idée qu'on risque un peu moins une atteinte aux personnes s'il y a une clôture. Mais je pense que c'est illusoire, personne dans le fond ne se sent protégé à $100 \%$. 


\section{Conclusion}

Il est clair que les logiques sécuritaires occupent une place très ambiguë dans les discours des promoteurs et des résidants, lorsque l'on interroge ces deux types d'acteurs sur cette nouvelle territorialité de l'habiter construite sur le mode de la fermeture et du contrôle des accès. D'un côté, nous ne pouvons qu'être frappés par la banalisation de la rhétorique sécuritaire dans le discours promotionnel. Certes, la systématisation, ou peu s'en faut, de la clôture et de la barrière dans la conception des programmes immobiliers neufs relève incontestablement d'une démarche marketing. Mais cette tendance ne fait que refléter la façon dont le couple insécurité/sécurité s'est imposé comme valeur centrale dans le débat de société aujourd'hui, avec le renouvellement de la question de l'insécurité en France à partir des années soixante-dix, accompagnant la montée des préoccupations insécuritaires et la tendance à l'uniformisation du discours sécuritaire entre la gauche et la droite.

Néanmoins, cette invasion de la rhétorique sécuritaire dans de multiples sphères de production de discours et de représentations (médiatique, politique, etc.) confirme la diversité des logiques sécuritaires à l'œuvre dans le mouvement de fermeture des espaces résidentiels en France. La confrontation entre le discours des promoteurs et celui des résidants d'ensembles fermés l'illustre, car si les premiers ont intégré la variable sécuritaire dans la conception même de leurs programmes, estimant ainsi répondre à la demande des ménages, ceux-ci portent un regard beaucoup plus distancié, varié et ambigu vis-à-vis de la prolifération des dispositifs de sécurisation dans l'habitat. Derrière ce regard pluriel, au-delà d'une quête chimérique de sécurité, se profile d'abord et avant tout une recherche de maîtrise de l'environnement résidentiel, qui renvoie à une volonté de contrôler son « chez-soi », d'assurer sa tranquillité.

\section{Bibliographie}

Ackermann (W.), Dulong (R.), Jeudy (H.-P.), 1983. - Imaginaires de l'insécurité, Paris, Librairie des Méridiens, $122 \mathrm{p}$.

Billard (G.), Chevalier (J.), Madoré (F.), 2005. - Ville fermée, ville surveillée. La sécurisation des espaces résidentiels en France et en Amérique du Nord, Rennes, PUR, 230 p.

Billard (G.), Chevalier (J.), Madoré (F.), Taburet (A.), Vualllat (F.), Raulin (F.), 2009. - « Typologie et représentations des ensembles résidentiels fermés ou sécurisés en France », Les Cahiers de la sécurité, $n^{\circ} 8$, p. 63-73.

Blakely (E. J.), Snyder (M. G.), 1997. - Fortress America: Gated Communities in the United States, Cambridge, Washington (DC), Brooking Institution Press, Lincoln Institute of Land Policy, 208 p.

Bonelli (L.), 2008. - La France a peur, Paris, La Découverte, 420 p.

Capron (G.) (dir.), 2006. - Quand la ville se ferme. Quartiers résidentiels sécurisés, Paris, Bréal, 288 p.

Charmes (É.), 2005. - La vie périurbaine face à la menace des gated communities, Paris, L'Harmattan, $219 \mathrm{p}$.

Glasze (G.), Webster (C.), Frantz (K.) (dir.), 2005. - Private Cities. Global and Local Perspectives, Londres, Routledge, $242 \mathrm{p}$.

Golovtchenko (N.), Souchet (F.), 2005. - « Des gated communities à la française? Les résidences fermées toulousaines », dans Haumont (B.), Morel (A.) (dir.), La société des voisins, Paris, Éditions de la Maison des Sciences de l'Homme, p. 145-167.

Le Goix (R.), Webster (C.), 2008. - « Gated Communities », Geography Compass, 2/4, p. 1189-1214.

Madoré (F.), 2003. - « Logiques géographiques et sociales de l'enclosure des espaces résidentiels en France », Belgeo, $\mathrm{n}^{\circ}$ 1, p. 99-113.

Mucchielli (L.), 2001. - Violences et insécurité. Fantasmes et réalités dans le débat français, Paris, La Découverte, $142 \mathrm{p}$. 
PAQuot (T.) (dir.), 2009. - Ghettos de riches. Tour du monde des enclaves résidentielles sécurisées, Paris, Perrin, $296 \mathrm{p}$.

Rey (H.), 2002, «La sécurité dans le débat politique », dans Mucchielli (L.), Robert (P.) (dir.), Crime et sécurité, l'état des savoirs, Paris, La découverte, p. 25-32.

Robert (P.), Ротtier (M.-L.), 1997, «Sur l'insécurité et la délinquance », Revue française de science politique, volume $47, n^{\circ} 5$, p. 630-644.

Vuaillat (F.), Madoré (F.), 2008. - « La parole habitante au sein d'une résidence fermée : le Newton à Carquefou », Cahiers Nantais, n 1, p. 41-50.

Wieviorka (M.), 1999, Violence en France, Paris, Seuil, 352 p.

Cet article a été reçu le 16 janvier 2009 et définitivement accepté le 26 juin 2009. 